PROCEEDINGS OF THE

AMERICAN MATHEMATICAL SOCIETY

Volume 127, Number 6, Pages 1723-1727

S 0002-9939(99)05209-0

Article electronically published on February 11, 1999

\title{
EXTREMAL POINTS OF A FUNCTIONAL ON THE SET OF CONVEX FUNCTIONS
}

\author{
T. LACHAND-ROBERT AND M. A. PELETIER \\ (Communicated by Jeffrey B. Rauch)
}

\begin{abstract}
We investigate the extremal points of a functional $\int f(\nabla u)$, for a convex or concave function $f$. The admissible functions $u: \Omega \subset \mathbf{R}^{N} \rightarrow \mathbf{R}$ are convex themselves and satisfy a condition $u_{2} \leq u \leq u_{1}$. We show that the extremal points are exactly $u_{1}$ and $u_{2}$ if these functions are convex and coincide on the boundary $\partial \Omega$. No explicit regularity condition is imposed on $f, u_{1}$, or $u_{2}$.

Subsequently we discuss a number of extensions, such as the case when $u_{1}$ or $u_{2}$ are non-convex or do not coincide on the boundary, when the function $f$ also depends on $u$, etc.
\end{abstract}

\section{INTRODUCTION}

The aim of this paper is to characterize the solutions of the variational problems

$$
\inf _{u \in \mathcal{C}} \int_{\Omega} f(\nabla u) \quad \text { or } \quad \sup _{u \in \mathcal{C}} \int_{\Omega} f(\nabla u)
$$

on a set of admissible functions

$$
\mathcal{C}=\left\{u: \bar{\Omega} \rightarrow \mathbf{R} \text { is convex and } u_{2} \leq u \leq u_{1}\right\},
$$

where $\Omega$ is a bounded subset of $\mathbf{R}^{N}$ and $u_{1}, u_{2}: \bar{\Omega} \rightarrow \mathbf{R}^{N}$ are convex and coincide on $\partial \Omega$ (see Section 2 for generalizations). The main theorem, Theorem 1, establishes a connection between the convexity properties of $f$ and the nature of the extremal points.

This sort of problem, and in particular the convexity constraint on $u$, is relatively rare in calculus of variations. However, certain very old problems appear to have this form, for instance Newton's problem of the body of minimal resistance (see [2] - here the function $f$ is neither convex nor concave). Also, in economy some interesting new problems also are stated with convexity constraint; we refer the reader to [6] and the references here for this type of applications.

For the integral in (1) to be well-defined on $\mathcal{C}$ we need to introduce some hypotheses. We recall that any convex function on $\Omega$ is Lipschitz continuous on compact

Received by the editors September 10, 1997.

1991 Mathematics Subject Classification. Primary 49K99.

Key words and phrases. Extremal points, convexity constraint, non-convex minimization.

Part of this work was carried out during a visit of the second author to Université Pierre et Marie Curie under the contract of the European Union 921 CHRX CT 94.

(C)1999 American Mathematical Society 
subsets of $\Omega$ and differentiable almost everywhere in $\Omega$; under the additional hypothesis

$$
\nabla u_{2} \text { is bounded a.e. on } \Omega \text {, }
$$

combined with $\left(H_{2}\right)$ below, the set $\mathcal{C}$ is embedded in $W^{1, \infty}(\Omega)$. Indeed, $\left(H_{2}\right)$ and the convexity imply that

$$
\|\nabla u\|_{L^{\infty}(\Omega)} \leq\left\|\nabla u_{2}\right\|_{L^{\infty}(\Omega)} \quad \forall u \in \mathcal{C} .
$$

Under the assumptions of Theorem 1 the function $f$ is continuous and therefore the integral is well-defined and bounded from below on $\mathcal{C}$.

We also remark here that by the results of [5] the set $\mathcal{C}$ is compactly imbedded in $W_{\text {loc }}^{1, p}(\Omega)$ for any $1 \leq p<\infty$. Consequently the infimum in (3) is attained. For a complete discussion on the continuity properties of functionals of this form, even without any assumption on the convexity of the functions, we refer the reader to [5].

For our application, the problem reduces to

$$
\min _{u \in \mathcal{C}} \int_{\Omega} f(\nabla u)
$$

where $f$ is convex or concave; the supremum is obtained by changing $f$ to $-f$.

Theorem 1. Let $u_{1}, u_{2}$ be convex and satisfy

$$
u_{1}=u_{2} \quad \text { on } \quad \partial \Omega .
$$

1. if $f$ is strictly convex, then $u=u_{1}$ is the unique solution of Problem (3);

2. if $f$ is strictly concave, then $u=u_{2}$ is the unique solution of Problem (3).

Remark. Except for the convexity constraint on the functions $u$, part 1 of this theorem corresponds to a classical problem in the calculus of variations, which is usually analysed using the Euler-Lagrange equation and methods from PDE theory. The convexity constraint prompts us to use different techniques, which has some interesting consequences for the regularity of the functions involved. First, we do not need to suppose any special regularity on the function $f$; in particular, $f$ need not be $C^{1}$. Second, Theorem 1 shows that the convexity constraint eliminates part of the regularizing effect that is commonly present in convex variational problems: the function $u_{1}$, a unique minimizer if $f$ and $u_{1}$ are both convex, need not be smooth; if $u_{1}$ is not convex (see generalizaton 2 below), then the minimizer $u_{1}^{* *}$ need not be smooth either, even in points where $u_{1}$ and $u_{1}^{* *}$ do not coincide.

Proof. Denote the integral in (3) by $F(u)$. We first prove the following lemma, adopting the notation $u \vee v=\max \{u, v\}$. We recall that if $u$ and $v$ are convex, then $u \vee v$ is, also.

Lemma 1. Suppose that $f$ is strictly convex. Let $u \in \mathcal{C}$ and suppose that $\theta: \Omega \rightarrow \mathbf{R}$ is an affine function such that the set $\omega=\{x \in \bar{\Omega}: \theta(x)>u(x)\}$ is non-empty. Then $F(u \vee \theta)<F(u)$.

Proof of the lemma. Since $\theta \vee u$ is equal to $\theta$ in $\omega$ and equal to $u$ in $\Omega \backslash \omega$, we have

$$
\delta F:=F(\theta \vee u)-F(u)=\int_{\omega}[f(\nabla \theta)-f(\nabla u)] .
$$

Define

$$
g(\xi)=f(\xi+\nabla \theta)-f(\nabla \theta)-f^{\prime}(\nabla \theta) \cdot \xi, \quad \forall \xi \in \mathbf{R}^{N},
$$


where for $f^{\prime}(\nabla \theta)$ we take any element of the subdifferential of $f$ at the point $\nabla \theta$ (remark that $\nabla \theta$ is constant on $\omega$ ). Writing $\varphi=u-\theta$, we have

$$
\delta F=-\int_{\omega} g(\nabla \varphi)
$$

since $\varphi=0$ on $\partial \omega$ implies $\int \nabla \varphi=0$.

The strict convexity of $f$ is passed on to $g$; by construction we also have

$$
g(0)=0=\inf _{\xi \in \mathbf{R}^{N}} g(\xi),
$$

which implies $g(\xi)>0$ for any $\xi \neq 0$. Hence $\delta F<0$ which concludes the proof of the lemma.

We now return to the proof of the theorem. Let us first assume that $f$ is strictly convex. Then, using the lemma, we obtain $F(\theta \vee u) \leq F(u)$ for any $u \in \mathcal{C}$ and any affine function $\theta$, the inequality being strict if $\theta \vee u \not \equiv u$ in $\Omega$.

In particular, if $v$ is any given function in $\mathcal{C} \backslash\left\{u_{1}\right\}$, there exists $\theta_{1}$ such that

$$
v \leq v_{1}:=v \vee \theta_{1} \leq u_{1} \quad \text { and } \quad v \not \equiv v_{1} \quad \text { in } \Omega .
$$

Also, since $v_{1}$ and $u_{1}$ are convex functions satisfying $v_{1} \leq u_{1}$, there exists a finite or infinite number of affine functions $\left(\theta_{k}\right)_{k>2}$ such that the sequence $\left(v_{k}\right)_{k>2}$ defined by $v_{k}:=\theta_{k} \vee v_{k-1}$ satisfies $v_{k} \leq u_{1}$ and $v_{k} \rightarrow u_{1}$ in the uniform topology (and therefore in $W^{1, p}(\Omega)$, since the functions $v_{k}$ are convex).

Consequently we have the sequence of inequalities

$$
F(v)>F\left(v_{1}\right) \geq F\left(v_{2}\right) \geq \cdots \geq F\left(v_{k}\right) \rightarrow F\left(u_{1}\right),
$$

hence $F(v)>F\left(u_{1}\right)$ and this concludes case 1 of the theorem, since $v$ is arbitrary.

If $f$ is concave, $-f$ is convex. Hence, using the lemma again, we get $F(\theta \vee u) \geq$ $F(u)$ for any $u \in \mathcal{C}$ and any affine $\theta$, the inequality being strict if $\theta \vee u \not \equiv u$ in $\Omega$.

If $v$ is any function in $\mathcal{C} \backslash\left\{u_{2}\right\}$, we can again define a finite or infinite sequence $\left(\theta_{k}\right)_{k>1}$ such that $v_{1}:=u_{2} \vee \theta_{1}$ satisfies $u_{2} \leq v_{1} \leq v$ and $u_{2} \neq v_{1}$, and if $v_{k}:=$ $v_{k-1} \vee \theta_{k}$, the sequence $\left(v_{k}\right)$ converges to $v$. We now have

$$
F\left(u_{2}\right)<F\left(v_{1}\right) \leq F\left(v_{2}\right) \leq \cdots \leq F\left(v_{k}\right) \rightarrow F(v)
$$

and therefore $F\left(u_{2}\right)<F(v)$. This concludes the proof of the theorem.

\section{Generalizations And improvements}

We have given our main theorem in a simple and convenient form, but many generalizations and improvements are possible. We give some of them here, and also discuss the accuracy of some of the hypotheses.

1. If $u_{1}$ is not convex, the conclusion of the theorem is unchanged if $f$ is concave, and should be changed to ' $u_{1}^{* *}$ is the unique solution' if $f$ is convex. Here $u_{1}^{* *}$ is the convex regularization of $u_{1}$ (also called the $\Gamma$-regularization; [3], Proposition 4.1). This is obvious since this function is the maximal element in $\mathcal{C}$ :

$$
u_{1}^{* *}(x)=\max \{u(x) ; u \in \mathcal{C}\}, \quad \forall x \in \Omega,
$$

and we can therefore replace $u_{1}$ by $u_{1}^{* *}$ without loss of generality. 
2. If $u_{2}$ is not convex, the situation is slightly more complicated if $f$ is concave (though the result remains if $f$ is convex). Indeed, in that case, it is clear from the proof that any solution $u_{0}$ of problem (3) must be 'minimal' in the following sense: for all $u \in \mathcal{C} \backslash\left\{u_{0}\right\}$, there exists $x \in \Omega$ such that $u(x)>u_{0}(x)$. On the other hand, many 'minimal' points can exist in $\mathcal{C}$, and there is no obvious way to decide which one minimizes $F$; it is also possible that more than one do. This situation is very similar to the usual problem of minimizing a concave function on a convex set.

3. It is not necessary for $f$ to be strictly convex or concave, except that if it is not, the minimizer is usually not unique (but $u_{1}$ or $u_{2}$ are minimizers as stated in the theorem; this can be easily proved by using an approximation argument on $f$, for instance). The extreme case is obtained when $f$ is affine; here $F$ is constant on the set $\mathcal{C}$, since $u=u_{1}$ on $\partial \Omega$ implies $\int \nabla u=\int \nabla u_{1}$.

If $f$ is neither convex nor concave, as happens for the Newton functional for instance, the situation is much more complicated. We refer the reader to [2] for the statement of the problem, and to [1] for an interesting nonsymmetry result. A first contribution towards a complete characterization of the solution is to be given in [4].

4. One can also consider $f$ depending also on $u$, for instance by minimizing $\int f(u, \nabla u)$ on $\mathcal{C}$. A simple generalization of the main theorem is as follows:

Lemma 2. Assume hypothesis $\left(\mathrm{H}_{2}\right)$ and consider the problem

$$
\inf _{u \in \mathcal{C}} \int_{\Omega} f(u, \nabla u)
$$

Then,

(a) if $f(u, p)$ is nondecreasing with respect to $u$ and convex with respect to $p$, then $u=u_{1}$ is a minimizer;

(b) if $f(u, p)$ is nonincreasing with respect to $u$ and concave with respect to $p$, then $u=u_{2}$ is a minimizer;

In both cases, the minimizer is unique if $f(u, p)$ is strictly monotone with respect to $u$ or strictly convex or concave with respect to $p$.

This follows directly with the same proof, since Lemma 1 is still true in these cases.

5. $\Omega$ may be unbounded; in that case, condition $\left(H_{2}\right)$ should be changed to either

$$
\lim _{x \in \Omega, x \rightarrow \infty}\left|u_{1}(x)-u_{2}(x)\right|=0,
$$

which is the natural generalization of $\left(H_{2}\right)$, or

$$
\lim _{x \in \Omega, x \rightarrow \infty}\left|\nabla u_{2}(x)\right|=+\infty .
$$

If this last condition is satisfied, then for any affine function $\theta$ and any $u \in \mathcal{C}$, the set $\{u<\theta\}$ is bounded so the proof is similar.

6. The hypothesis $\left(H_{2}\right)$ is crucial, since for instance, if we just assume $u_{1}$ and $u_{2}$ to be affine functions, one may have either $u_{1}>u_{2}$ or $u_{1}<u_{2}$ in $\Omega$ just by adding a constant to one of them; but adding a constant to $u$ does not change $F(u)$, hence there is no way to compare $F\left(u_{1}\right)$ and $F\left(u_{2}\right)$ in this example. (Many thanks to A. Damlamian for this counterexample.) 
Hence, if $u_{1} \neq u_{2}$ on some part of $\partial \Omega$, a minimizer $u$ different from $u_{1}$ and $u_{2}$ could exist in $\mathcal{C}$. It is still possible to give some information if $f$ is strictly convex. Since the inequality of $u_{1}$ and $u_{2}$ on the boundary implies that $\nabla u$ is not bounded on $\mathcal{C}$, we need to impose restrictions on $f$ in order to ensure that $F(u)$ is finite for all $u \in \mathcal{C}$. One can suppose that $f$ is bounded from below; but it appears that the condition

$$
\exists c_{1}, c_{2}>0, \forall \xi \in \mathbf{R}^{N}, \quad f(\xi)>-c_{1}-c_{2}|\xi|
$$

is sufficient even to ensure $\inf F>-\infty$, since for any convex function $u$,

$$
\int_{\Omega}|\nabla u| \leq 2 N \operatorname{diam}(\Omega)^{N-1} \operatorname{osc}_{\bar{\Omega}} u
$$

where we adopt the notation $\operatorname{osc} u=\sup u-\inf u$. This follows from considering the integral of $\left|\partial u / \partial x_{1}\right|$ in the direction of $x_{1}$. Since $u$ is convex the oscillation of $u \in \mathcal{C}$ is bounded by $\sup u_{1}-\inf u_{2}$.

Lemma 3. Assume that $f$ is strictly convex, but hypothesis $\left(H_{2}\right)$ is not satisfied. Then for any minimizer $u$ of $F$ on the set $\mathcal{C}$ and any given point $x \in \Omega$, either $u(x)=u_{1}^{* *}(x)$ or for any affine function $\theta$ satisfying $\theta(x)=u(x)$ and $\theta \leq u$ we have

$$
\exists y \in \partial \Omega, \quad u_{1}(y)>\theta(y)>u_{2}(y) .
$$

This lemma generalizes the main theorem (for $f$ convex) and can be proved the same way: just observe that if condition (5) is not satisfied, then there exists an $\epsilon>0$ such that the affine function $\theta+\epsilon$ satisfies the conditions of Lemma 1 with respect to $u$, hence $u$ is not minimal.

\section{REFERENCES}

[1] F. Brock, V. Ferone and B. Kawohl, A symmetry problem in the calculus of variations, Calculus of Variations and Partial Differential Equations, 4 (1996), pp. 593-599. MR 97i:49002

[2] G. Buttazzo, V. Ferone and B. Kawohl, Minimum problems over sets of concave functions and related questions, Math. Nachrichten, 173 (1993), pp. 71-89. MR 96b:49005

[3] I. Ekeland and R. Temam, Convex Analysis and Variational Problems, North-Holland (1972). MR 57:3931b

[4] T. Lachand-Robert and M. A. Peletier, An example of non-convex minimization and an application to Newton's problem of the body of least resistance, in preparation.

[5] P. Marcellini, Nonconvex Integrals of the Calculus of Variations, Proceedings of 'Methods of Nonconvex Analysis', Varenna 1989, ed. A. Cellina, Lecture Notes in Math., 1446, Springer (1990), pp. 16-57. MR 91j:49002

[6] J.-C. Rochet and P. Choné, Ironing, Sweeping and Multidimensional screening, to appear in Economica.

Université Pierre et Marie Curie, Laboratoire D’Analyse Numérique, 75252 Paris Cedex 05, France

E-mail address: lachand@ann.jussieu.fr

University of Bath, Claverton Down, Bath BA2 7AY, United Kingdom

E-mail address: M.A.Peletier@bath.ac.uk 